Contract No. and Disclaimer:

This manuscript has been authored by Savannah River Nuclear Solutions, LLC under Contract No. DE-AC09-08SR22470 with the U.S. Department of Energy. The United States Government retains and the publisher, by accepting this article for publication, acknowledges that the United States Government retains a non-exclusive, paid-up, irrevocable, worldwide license to publish or reproduce the published form of this work, or allow others to do so, for United States Government purposes. 


\title{
SRNL-STI-2011-00373
}

\section{The Effect of Various Detector Geometries on the Performance of CZT using One Crystal}

\author{
Aaron L. Washington, II,* Lucile C. Teague, and Martine C. Duff \\ Savannah River National Laboratory, Aiken, SC 29808 \\ Arnold Burger, Michael Groza, and Vladimir Buliga \\ Fisk University, Nashville, TN 37208-3051
}

Keywords: CZT; Detector Geometry; Gamma Radiation Source; Detector Performance

Email: aaron.washington@srnl.doe.gov

\begin{abstract}
CdZnTe (CZT) continues to be a major thrust interest mainly due to its potential application as a room temperature radiation detector. The performance of CZT detectors is directly related to the charge collection ability which can be affected by the configuration of the electrical contact. The charge collection efficiency is determined in part by the specific geometry of the anode contact which serves as the readout electrode. In this report, contact geometries including single pixel, planar, coplanar, and dual anode will be systematically explored by comparing the performance efficiencies of the detector using both low and high energy gamma rays. To help eliminate the effect of crystal quality variations, the contact geometries were fabricated on the same crystal detector with minimal polishing between contact placements.
\end{abstract}

\section{Introduction}

$\mathrm{CdZnTe}(\mathrm{CZT})$ detectors have been introduced as gamma- and X-ray detectors with significant advances over current industrial detection techniques, such as silicon and germanium detectors, because of their high atomic number and room temperature 


\section{SRNL-STI-2011-00373}

operation ability. ${ }^{1,2}$ The bulk and surface properties of CZT are often relevant to its various applications. ${ }^{3}$ The major factors that influence detector performance include the geometry of the contact and thickness of a detector as well as crystalline quality and defect content. To optimize the energy resolution for gamma detectors, previous studies have focused on the electron charge transport properties of the material, crystal uniformity, and the contact geometry aimed at resolving the effects of hole trapping. ${ }^{4}$ Electron transport properties and crystal uniformity are mainly dictated by the type of detector (i.e. silicon, high purity germanium (HPGe), CZT), in addition to the growth process (i.e. traveling heater method (THM), modified vertical Bridgman (MVB), physical vapor transport, etc.) that was used to produce the low defect materials.

The room temperature dark current of a useful CZT spectrometer is on the order of a few $\mathrm{nA}$ which corresponds to a concentration of $10^{4}-10^{6}$ carriers $\mathrm{cm}^{-3}$. When an ionizing particle interacts with a CZT detector, additional electron and hole carriers are generated. As the electrons move through the detector, an electric charge will be induced on the collecting anode. ${ }^{5}$ However, a fraction of the electron density that is driven to the surface can be lost due to the high trapping rate at the CZT surface. ${ }^{6,7}$ With good charge carrier mobility, a highly resistive surface with a contact will efficiently collect the signal from the detector. The charge collection at the anode contact is significantly altered on a single crystal detector by simply changing the geometry of the gold $(\mathrm{Au})$ contact on the crystal face. $^{8,9,10,11}$

The geometries that are currently used to produce highly energy efficient detectors include planar, ${ }^{1}$ coplanar grid, ${ }^{12,13,14,15,16,17}$ single pixel, ${ }^{1,18}$ and dual anode. ${ }^{5,19}$ Because the geometry of the contacts directly affects the collection of electrons at the 


\section{SRNL-STI-2011-00373}

surface, it also provides some insight into the electron transport properties of the material. The contact acts as a guide to direct the electron flow toward a particular part of the surface if so desired. There is a significant difference in the carrier collection based on geometry used in addition to the dependence of the carrier mobility on the homogeneity of the crystal. Additionally, the separate components of the contact geometries including ground or dead voltage, pixels, and/or guard rings influence how the surface leakage current affects the overall performance of the detector. ${ }^{13}$

Gold is an ideal material for use with non-injecting and non-blocking contacts on semi-insulating CZT during room temperature operation. Additionally, the work functions of Au and CZT are nearly identical which affects the ability of charge carriers to flow through the material. ${ }^{7}$ The geometry of the Au contact also allows for various regions of collection on the anode face of the crystal. Finally, the performance depends on how the internal electric field is distributed and collected at the contact.

In this report, we present a systematic study of the detector performance using four different detector geometries on a single CZT crystal. A systematic study was conducted using multiple geometries re-fabricated on the same CZT crystal. We performed minimal polishing between fabrications to help maintain similar crystal thicknesses and surface properties for all geometries. The use of one CZT crystal helps to eliminate any discrepancies in performance that could arise due to differences in internal uniformity and crystal size independent to each detector. To our knowledge, this is the first systematic study showing the performance differences associated with using various contact geometries for both low and high energy gamma sources. These geometries include planar, coplanar, single pixel, and dual anode using Au contacts 


\section{SRNL-STI-2011-00373}

deposited using standard photolithography and sputtering techniques on "as polished" crystals with no etching. The same crystal was used for all geometries where the crystal was polished and the Au contacts were re-deposited onto the surface in between measurements.

\section{Experimental}

The CZT sample used in this study was grown using the THM with an incorporation of $10 \% \mathrm{Zn}$ as described previously ${ }^{20,21}$ and was provided by Redlen Technologies (Victoria, B.C., Canada). The CZT crystal measured $10 \times 10 \times 2.1 \mathrm{~mm}^{3}$ and is hereby referred to as "Redlen 64039B.,"22 The sample was polished with standard techniques down to a fine polish with $0.05 \mu \mathrm{m}$ alumina. Au contacts were sputtered onto the crystal and testing was performed using ${ }^{241} \mathrm{Am}(59.5 \mathrm{keV})$ as a low energy gamma source and ${ }^{137} \mathrm{Cs}(662 \mathrm{keV})$ as a high energy gamma source.

The geometries of the contacts include single pixel, planar, coplanar, and dual anode as shown in Figure 1. The pixel geometry has a diameter of $2.7 \mathrm{~mm}$ with a 150 $\mu \mathrm{m}$ gap between the pixel and guard. The planar geometry was a uniform layer of Au applied to the entire surface of the crystal. The coplanar geometry used 14 Au strips with a width of $315 \mu \mathrm{m}$ and inter-strip gaps of $195 \mu \mathrm{m}$ in an inter-digitized "comb-like" system. Also, Au wires were placed at opposite corners to attach to the electrical probes to prevent damage to the contact using an epoxy adhesive to connect the wires to the surface. The dual anode geometry used two parallel Au strips on the anode face. The centered $\mathrm{Au}$ strips are $0.2 \mathrm{~mm}$ wide with a $0.2 \mathrm{~mm}$ gap between them to provide the most

efficient collection. ${ }^{19}$ For both the coplanar and dual anode geometries, there was a secondary DC power supply providing a differential bias of $50 \mathrm{~V}$ used across the 


\section{SRNL-STI-2011-00373}

alternating coplanar strips and on the readout anode contact to focus the electron collection to the specified electrode. The shaping time for all measurements was maintained at $2 \mu \mathrm{s}$ to eliminate the effect of curve shaping on peak resolution. The cathode for all configurations was a uniform layer of $\mathrm{Au}$ on the crystal face opposite the anode.

The peak resolution was obtained by dividing the full-width at half maximum (FWHM) of the spectral peak by the selected peak energy of the gamma source. The performance data for the detector was obtained via an Amptek A250 CF charge sensitive preamplifier (Oak Ridge, TN) in addition to an Ortek (Oak Ridge, TN) amplifier. These instruments were coupled to a multi-channel analyzer made by Camberra (Meriden, CT). A LeCroy DA 1855A differential amplifier was used in the coplanar and dual anode geometries.

\section{Results and Discussion}

\section{Low Energy Gamma Detection}

The use of single crystals as gamma ray detectors shows a significant energy dependence on the performance of each detector based on its size and its individual uniformity. Traditionally, higher energy (662 keV) gamma emitters like ${ }^{137} \mathrm{Cs}$ are more often studied in single crystal detectors than low energy (59.5 keV) emitters like ${ }^{241} \mathrm{Am}^{17}$ and in general, high gamma energy detection is largely determined by material uniformity due to the deeper penetration ability of the higher energy gamma radiation. In contrast, the low energy gamma radiation has very shallow penetration depths and consequently require more iterations (longer scan times) to overcome the detector signal to noise contribution. ${ }^{23}$ For this reason, it is necessary to analyze radiation detectors for 


\section{SRNL-STI-2011-00373}

both low and high energy sources to report their overall effectiveness. In addition, the difference in the charge collection area that is available via different detector geometries impacts the collection efficiency of a specific radiation source. This observation was based both on the energy of the emission peak, which can range widely throughout the gamma energy spectrum, and the width of the detector. In addition, the different effects of surface and side leakage current contributions on detector performance from with detector geometry are considered.

The geometry and gamma radiation source energy impact the collection efficiency and, subsequently, the performance of the detector. To assess the collection response using a low energy gamma source $\left({ }^{241} \mathrm{Am}\right)$ shown in Figure 2, we compare the FWHM as a function of bias voltage for the four geometries. The single pixel geometry is the most efficient detector geometry for this particular crystal with an average peak width of 2.425 $\mathrm{keV}(4.1 \%)$ across the entire bias range. The planar contact has a peak width of 3.304 $\mathrm{keV}(5.5 \%)$ across the bias range which is still a very good detector. Both the dual anode and the coplanar detectors are relatively poor in their collection efficiencies with peak width averages of $7.407 \mathrm{keV}(12.4 \%)$ and $15.207 \mathrm{keV}(25.5 \%)$ for the dual anode and coplanar geometries, respectively. The rapid decrease in peak resolution for both dual anode and coplanar geometries as compared to pixel and planar geometries is unexpected and the decreased resolution is assumed to be due to the sharp increase of surface contributions with the increased exposed surface. In addition, the coplanar grid and dual anode geometries represent detectors where two anode channels are used. They exhibit difficulties in localization of charge and diminished collection efficiency due mainly to 


\section{SRNL-STI-2011-00373}

increased surface contributions. This combination results in decreased energy resolution as compared to other geometries. ${ }^{5}$

\section{High Energy Gamma Detection}

In general, solid state detectors of CZT will resolve high energy gamma radiation better than low energy gamma radiation due to poor hole transport and moderate electron trapping that occurs at low energy. ${ }^{24,14}$ However, using electron-only detection techniques (i.e. pixels and coplanar grids) allow for highly efficient charge collection in uniform crystals. $^{25,26}$ In the literature, the majority of detector studies presented compare the capabilities of electron only detectors using high energy ${ }^{137} \mathrm{Cs}$ sources as a standard for measuring energy resolution. ${ }^{27,28}$

High energy radiation interacts with the entire volume of the crystal. It requires a highly homogeneous material to prevent charge trapping (at the surface and in the bulk), which decreases the resolution of the energy peak. High energy gamma spectrometer responses from ${ }^{137} \mathrm{Cs}$ are shown in Figure 3 where the line spectra illustrate the difference in the performance of different contact geometries. The small single pixel geometry has the best resolution of ${ }^{137} \mathrm{Cs}$ with a peak width of $13.44 \mathrm{keV}(2.0 \%)$. Both dual anode and coplanar geometries have a significant decrease in the high energy gamma resolution when acquired under identical experimental conditions. The dual anode geometry has a peak width of $36.81 \mathrm{keV}(5.5 \%)$. The coplanar grid geometry has a peak width of 51.23 $\mathrm{keV}(7.7 \%)$. The diminished performance of these geometries clearly illustrates the increase in the surface contribution. This is evidenced by the decreased performance in geometries of relatively greater exposed surface area, such as the coplanar and dual anode geometries. However, both coplanar grid and dual anode detectors are highly effective in 


\section{SRNL-STI-2011-00373}

minimizing data acquisition and analysis complexity relative to that of most single pixel detectors. $^{5}$

Contrary to those low-energy response results that we report above (in which the best performance was observed for the single pixel geometry), we find that the opposite is true for high energy gamma detection. For high energy gamma detection, the planar geometry had the worst resolution. The peak width could not be determined or measured without the introduction of significant amounts of error. The different geometries were monitored by changes in the FWHM of the detector. This provides insight into the mechanism by which surface geometry contributes to the observed changes in detector performance.

\section{Conclusions}

This work compiles the results from our testing of four different contact geometries using the same CZT detector crystal and the same electronics throughout. The tabulated form of the collected data is shown in Table 1. One of the most important results from this study is the observed difference between the high energy response from

${ }^{137} \mathrm{Cs}$ and the lower energy response from ${ }^{241} \mathrm{Am}$. Our results highlight several important issues for consideration when evaluating performance and making the determination for the optimal configuration for a detector. One would expect a disparity in collection efficiencies between contact geometries due to the varying charge collection efficiencies and surface contributions. However, in our experiments the extreme difference in performance of certain anode geometries was unexpected due to the fact that the same crystal was used throughout, which helped eliminate potential discrepancies in performance due to variations in crystal quality. Our study determined that the best 


\section{SRNL-STI-2011-00373}

overall contact geometry for both low and high gamma sources is the single pixel geometry. The contact geometry with a very poor performance was the widely used coplanar. Its performance was low in the low energy region and not as well as dual anode and single pixel geometries in the high energy region. With respect to geometry versus performance at certain energies, this work provides some insight into choosing contact geometries. The best geometry must be highly efficient throughout the full energy spectrum of interest. It must also have the best performance and energy resolution. This approach is important to the assessment of candidate CZT material for potential radiation spectrometer use.

\section{Acknowledgements}

This document was prepared in conjunction with work accomplished under Contract No. DE-AC09-08SR22470 with the U.S. Department of Energy (US DOE). This work was supported by US DOE—National Nuclear Security Administration, through the Office of Nonproliferation and Verification Research and Development-NA-22 (Grant No. DEFG52-05NA27035) and the National Science Foundation through the Fisk University Center for Physics and Chemistry of Materials (CPCoM), and Cooperative Agreement CA: HRD-0420516 (CREST program). We thank Redlen Technologies for supplying the crystal for our studies.

\section{Figure Captions}

Figure 1: This figure shows the four different detector geometries described in this manuscript including: single pixel (upper left), coplanar (bottom left), planar (upper right), and dual anode (bottom right). 


\section{SRNL-STI-2011-00373}

Figure 2: The detector performance of CZT using a ${ }^{241}$ Am source with four different surface geometries: coplanar (blue triangles), dual anode (green diamonds), planar (black squares), and small single pixel (red circles) on the anode contact. The plot displays a difference in FWHM as a function of voltage bias.

Figure 3: The ${ }^{137} \mathrm{Cs}$ detector performance line spectrum is displayed showing the difference between the resolving ability of four different geometries: dual anode (green dash dot dot dash line), single pixel (blue dash dot dash line), coplanar (red dash line), and planar (black solid line). The spectra are separated vertically for visual clarity only.

Table 1: The ${ }^{241} \mathrm{Am}$ and ${ }^{137} \mathrm{Cs}$ peak resolutions are tabulated in both FWHM (keV) and resolution $(\%)$ for the discussed geometries including planar, co-planar, single pixel, and dual anode.

\section{References}

1 T. E. Schlesinger, J. E. Toney, H. Yoon, E. Y. Lee, B. A. Brunett, L. Franks, R. B. James, Mater. Sci. Eng. R-Rep. 32, 103 (2001).

2 P. N. Luke, M. Amman, C. Tindall, J. S. Lee, J. Radioanal. Nucl. Chem. 264, 145 (2005).

3 L. C. Teague, S. A. Hawkins, M. C. Duff, M. Groza, V. Buliga, A. Burger, J. Electron. Mater. 38, 1522 (2009).

4 P. N. Luke, J. S. Lee, M. Amman, K. M. Yu, IEEE Trans. Nucl. Sci. 49, 1950 (2002).

5 J. W. Martin, A. B. Garson Iii, Q. Li, K. Lee, M. Groza, V. Buliga, A. Burger, H. Krawczynski, presented at the SPIE, San Diego, CA, USA, 2009 (unpublished).

6 A. E. Bolotnikov*, S. E. Boggs, C. M. H. Chen, W. R. Cook, F. A. Harrison, S. M. Schlinder, presented at the SPIE, 2000 (unpublished).

7 P. J. Sellin, G. Prekas, J. Franc, R. Grill, Appl. Phys. Lett. 96, 133509 (2010).

8 G. A. Carini, G. S. Camarda, Z. Zhong, D. P. Siddons, A. E. Bolotnikov, G. W. Wright, B. Barber, C. Arnone, R. B. James, J. Electron. Mater. 34, 804 (2005).

9 A.E. Bolotnikov, G.S. Camarda, G.A. Carini, Y. Cui, L. Li, R.B. James, Nucl. Instrum. Methods A 579, 120 (2007).

10 A. E. Bolotnikov, G. S. Camarda, Y. Cui, A. Hossain, G. Yang, H. W. Yao, R. B. James, Nuclear Science, IEEE Transactions on 56, 791 (2009).

11 G. A. Carini, A. E. Bolotnikov, G. S. Camarda, G. W. Wright, R. B. James, L. Li, Appl. Phys. Lett. 88, 143515 (2006).

P. N. Luke, M. Amman, J. S. Lee, H. Yaver, Nucl. Instrum. Methods Phys. Res. Sect. AAccel. Spectrom. Dect. Assoc. Equip. 439, 611 (2000). 


\section{SRNL-STI-2011-00373}

13

14

15

16

17

18

R. Gonzalez, J. M. Perez, O. Vela, E. de Burgos, J. C. Oller, V. Gostilo, IEEE Trans. Nucl. Sci. 52, 2076 (2005).

4 J. V. Dawson, C. Montag, C. Reeve, J. R. Wilson, K. Zuber, Nucl. Instrum. Methods Phys. Res. Sect. A-Accel. Spectrom. Dect. Assoc. Equip. 599, 209 (2009).

G. De Geronimo, A. E. Bolotnikov, G. Carini, J. Fried, P. O'Connor, S. A. Soldner, IEEE Trans. Nucl. Sci. 53, 456 (2006).

P. N. Luke, M. Amman, and J. S. Lee, IEEE Trans. Nucl. Sci. 51, 1199 (2004).

M. Amman, J. S. Lee, and P. N. Luke, IEEE Trans. Nucl. Sci. 53, 3035 (2006).

T. R. Bloxham and M. Freer, Nucl. Instrum. Methods Phys. Res. Sect. A-Accel. Spectrom. Dect. Assoc. Equip. 572, 722 (2007).

M. Groza, H. Krawczynski, A. Garson, J. W. Martin, K. Lee, Q. Li, M. Beilicke, Y. L. Cui, V. Buliga, M. S. Guo, C. Coca, A. Burger, J. Appl. Phys. 107, 023704 (2010).

M. Funaki, T. Ozaki, K. Satoh, R. Ohno, Nucl. Instrum. Methods Phys. Res. Sect. A-Accel. Spectrom. Dect. Assoc. Equip. 436, 120 (1999).

R. Triboulet, T. N. Duy, A. Durand, J. Vac. Sci. Technol. A-Vac. Surf. Films 3, 95 (1985).

S. A. Awadalla, J. Mackenzie, H. Chen, B. Redden, G. Bindley, M. C. Duff, A. Burger, M. Groza, V. Buliga, J. P. Bradley, Z. R. Dai, N. Teslich, D. R. Black, J. Cryst. Growth 312, 507 (2010).

P. N. Luke, M. Amman, J. S. Lee, P. F. Manfredi, IEEE Trans. Nucl. Sci. 48, 282 (2001).

4 A. Niemela, H. Sipila, V. I. Ivanov, Nucl. Instrum. Methods Phys. Res. Sect. A-Accel. Spectrom. Dect. Assoc. Equip. 377, 484 (1996).

5 M. Amman, J. S. Lee, P. N. Luke, H. Chen, S. A. Awadalla, R. Redden, G. Bindley, IEEE Trans. Nucl. Sci. 56, 795 (2009).

B. W. Sturm, Z. He, T. H. Zurbuchen, P. L. Koehn, IEEE Trans. Nucl. Sci. 52, 2068 (2005).

7 A. E. Bolotnikov, G. S. Camarda, G. A. Carini, Y. Cui, K. T. Kohman, L. Li, M. B. Salomon, R. B. James, IEEE Trans. Nucl. Sci. 54, 821 (2007).

28 Z. He and B. W. Sturm, Nucl. Instrum. Methods Phys. Res. Sect. A-Accel. Spectrom. Dect. Assoc. Equip. 554,291 (2005). 